Cristina Pellegrino Baena ${ }^{1}$

Ana Luísa Muccillo-Baisch²

Tabajara Lucas Almeida ${ }^{3}$

Clarice De La Rocha ${ }^{4}$

Ozéia Simões Franco ${ }^{5}$

Daniel Olmedo ${ }^{6}$

Maria Cristina Flores Soares ${ }^{7}$

\section{Impacto de um programa piloto de promoção da saúde para trabalhadores marítimos de rebocadores}

\author{
The impact of a pilot project on health promotion for \\ maritime tugboat workers
}

${ }^{1}$ Mestre em Ciências da Saúde pela Universidade Federal do Rio Grande (FURG), Rio Grande, RS, Brasil.

${ }^{2}$ Coordenadora e docente do Programa de Pós Graduação em Ciências da Saúde (PPGCiSau) e do Instituto de Ciências Biológicas (ICB) da Universidade Federal do Rio Grande (FURG), Rio Grande, RS, Brasil.

${ }^{3}$ Docente em Estatística na Universidade Federal do Rio Grande (FURG), Rio Grande, RS, Brasil.

${ }^{4}$ Nutricionista do Hospital Universitário da Universidade Federal do Rio Grande (FURG), Rio Grande, RS, Brasil.

${ }^{5}$ Educador Físico, mestrando do Programa de Pós-Graduação em Ciências da Saúde (PPGCiSau), da Universidade Federal do Rio Grande (FURG), Rio Grande, RS, Brasil.

${ }^{6}$ Docente da Faculdade de Medicina da Universidade Federal do Rio Grande (FURG), Rio Grande, RS, Brasil.

${ }^{7}$ Docente do Programa de Pós Graduação em Ciências da Saúde (PPGCiSau) e do Instituto de Ciências Biológicas (ICB) da Universidade Federal do Rio Grande (FURG), Rio Grande, RS, Brasil.

Contato:

Professora Doutora Maria Cristina Flores Soares

Secretaria PPGCiSau - 4º andar - Área Acadêmica Professor Newton Azevedo - Campus da Saúde.

Rua General Osório, s/n. Centro, Rio Grande/RS, Brasil.

CEP: 96200-400

E-mail:

mcflores01@gmail.com

\section{Resumo}

Objetivo: analisar um programa piloto de promoção de saúde com trabalhadores marítimos de rebocadores baseado no estímulo à prática de atividade física regular e no acompanhamento nutricional. Metodologia: fizeram parte do estudo 10 trabalhadores do porto do Rio Grande, sul do Brasil, que foram submetidos a um programa de 22 semanas, composto de atividade física e acompanhamento nutricional regular. Antes e após a intervenção foram realizadas as seguintes avaliações: física, bioquímica sanguínea, $\mathrm{VO}_{2}$ máximo e qualidade de vida (WHOQOL Bref). Na análise foram utilizados os testes t de Student pareado, o de Wilcoxon e o de Correlação de Pearson. Resultados: foi verificado diminuição na média de peso (84,3 vs. $82,1 \mathrm{~kg}$; $\mathrm{p}=0,03)$, da circunferência abdominal (97,1 vs. $91,7 \mathrm{~cm} ; \mathrm{p}=0,005)$ e do quadril $(103,5$ vs. 100,7 cm; $\mathrm{p}=0,005)$. O tempo de atividade física se correlacionou com a redução da circunferência abdominal $(\mathrm{r}=0,636 ; \mathrm{p}=0,048)$ e com o índice cintura-quadril $(\mathrm{r}=0,683 ; \mathrm{p}=0,03)$. Houve redução no número de trabalhadores com triglicerídeos alterados ( 7 vs. 3 ; p =0,04). A análise da qualidade de vida não apresentou alteração. Conclusão: o modelo de intervenção de promoção de saúde proposto mostrou, para os trabalhadores participantes, resultados positivos em parâmetros importantes para o risco cardiovascular.

Palavras-chave: promoção de saúde; saúde do trabalhador; estilo de vida; hábitos alimentares; exercício.

\section{Abstract}

Objective: To analyze a pilot study on health promotion for maritime tugboat workers. Methodology: Ten tugboat workers from Rio Grande harbor located in Southern Brazil participated in a 22 week program, consisting of physical activity and regular nutritional counseling. Physical tests, blood biochemistry, $\mathrm{VO}_{2}$ max, and test on quality of life (WHOQOL Bref) were applied before and after the intervention. Paired Student $t$ test, Wilcoxon test, and Pearson correlation were used. Results: Decrease on weight $(84.3$ x $82.1 \mathrm{~kg}$; $p=0.03)$, waist circumference $(97.1 \times 91.7 \mathrm{~cm} ; p=0.005)$ and hip circumference (103.5 x $100.7 \mathrm{~cm} ; p=0.005)$ was observed. Physical activity duration showed correlation with reduction of waist circumference $(r=0.636 ; p=0.048)$ and waist-hip ratio ( $r=0.683 ; p=0.03)$. The number of workers with elevated triglycerides was reduced ( 7 vs. $3 ; p=0.04$ ). Life quality results did not alter. Conclusion: The health promotion intervention model showed positive results in important parameters for cardiovascular risk among tugboat workers under study.

Keywords: health promotion; occupational health; life style; food habits; exercise. 


\section{Introdução}

O conceito de promoção de saúde vem se difundindo como estratégia eficaz para a manutenção de condições adequadas de saúde da população e a redução dos custos com a medicina especializada que se faz necessária quando as condições da doença já estão estabelecidas (BUSS, 2000).

No que se refere à saúde do trabalhador, o forte impacto socioeconômico da doença, não só sobre a empresa, mas também sobre a família do trabalhador e a sociedade de uma forma geral, tem despertado o interesse para o desenvolvimento de ações que estimulem a adoção, por parte desses trabalhadores, de práticas saudáveis que contribuam para a promoção de sua saúde e a melhora de sua qualidade de vida.

Neste sentido, as empresas, enquanto geradoras de recursos financeiros para os indivíduos, tornam-se locais privilegiados para adoção de ações de promoção e de redução de riscos à saúde de seus colaboradores. Em nível mundial, grandes empresas norte-americanas e europeias vêm adotando práticas de bem-estar de seus empregados desde o fim da década de 1980 (GRAÇA, 1999). Em consonância com este movimento, o Brasil registra o início destas práticas de promoção de saúde e qualidade de vida desde o fim da década de 1990.

A obesidade é um dos problemas que mais frequentemente afetam a saúde da população em geral e a dos trabalhadores de forma mais específica, transformando-se em uma questão de saúde pública de grande magnitude. Mais de $40 \%$ da população brasileira apresenta sobrepeso ou obesidade, e esta prevalência é maior a partir dos 45 anos, podendo chegar a $61 \%$. Outro dado alarmante para a saúde é que a proporção de inativos fisicamente pode chegar a $65 \%$ em algumas regiões do país (OGATA, 2007).

A epidemia de obesidade leva a um aumento na incidência de dislipidemias e doenças associadas à obesidade, como diabetes mellitus, doenças digestivas e hipertensão arterial sistêmica, sendo as doenças cardiocirculatórias a primeira causa de mortalidade na população adulta em nossa região (INSTITUTO BRASILEIRO DE GEOGRAFIA E ESTATÍSTICA, 2002). O excesso de peso corporal pode acarretar ainda sobrecarga ao sistem osteomuscular, gerando patologias crônicas como lombociatalgias, discopatias e tendinites diversas (WORLD HEALTH ORGANIZATION, 2000). Quando se considera a saúde dos trabalhadores, a obesidade afeta a qualidade de vida dos indivíduos, suas relações sociais, sua disponibilidade para o trabalho e consequentemente, sua capacidade produtiva (MENDONÇA; ANJOS, 2004).

O chamado "estilo de vida contemporâneo", caracterizado pelo aumento do fornecimento de energia pela dieta associado a uma redução da atividade física, faz desses fatores os verdadeiros determinantes da obesidade. Uma meta-análise recente mostrou o efeito benéfico sobre a saúde dos trabalhadores de intervenções que buscam modificar os "estilos de vida" e o consequente retorno econômico para as empresas com a realização de programas bem estruturados (CHAPMAN, 2002).

Uma das atividades fundamentais para o êxito da atividade portuária é o trabalho desenvolvido pelos trabalhadores marítimos de rebocadores. Esses trabalhadores auxiliam nas manobras de atracagem dos grandes navios em áreas portuárias e também participam de manobras dessas embarcações em alto mar. As atividades desempenhadas pelos marítimos de rebocadores são consideradas estressantes devido ao risco material e humano decorrente das próprias manobras, o que pode ser aumentado devido a mudanças nas condições climáticas. A tripulação é formada pelo mestre condutor do rebocador, pelos responsáveis pelas máquinas e pelos homens de convés. Esses trabalhadores exercem suas tarefas em turnos de trabalho de 72 horas, necessitando da presença de um cozinheiro a bordo para o preparo das refeições.

Um estudo sobre a mortalidade proporcional por doenças cardiovasculares em trabalhadores marítimos britânicos indica valores acima de 70\%, dos quais 58\% ocorrem a bordo (ROBERTS, 2003)

Considerando o nível de responsabilidade e a carga de estresse inerente à atividade desenvolvida pelos trabalhadores marítimos de rebocadores, foi identificada a necessidade de avaliar o perfil de saúde desta população e propor aos mesmos a participação em um programa de promoção da saúde baseado no estímulo à prática de atividade física regular e acompanhamento nutricional.

Este relato de experiência tem como objetivo apresentar os resultados obtidos com este programa piloto de promoção de saúde mostrando as mudanças observadas no perfil de saúde dos trabalhadores marítimos de rebocadores.

\section{Metodologia}

Fizeram parte deste programa piloto de promoção da saúde, 10 trabalhadores marítimos de rebocadores escolhidos entre um total de 53 que trabalhavam na empresa. A escolha desse grupo de marítimos deu-se pelo fato de constituirem as duas tripulações do rebocador com maior número de manobras no porto conforme calendário apresentado pela empresa.

No início das atividades e após 22 semanas de intervenção, foram coletados dados como idade, peso $(\mathrm{kg})$, altura $(\mathrm{cm})$ e medidas da circunferência da cintura (CA) e do quadril (CQ) (cm). Com base nesses dados, foi calculado o índice de massa corporal 
(IMC) $\left(\mathrm{kg} / \mathrm{m}^{2}\right)$ para verificar a adequação da situação nutricional e o índice cintura-quadril (ICQ).

Os trabalhadores realizaram avaliações bioquímicas do sangue tendo sido comparados os valores iniciais e finais dos seguintes parâmetros: triglicerídeos, colesterol total, fração de colesterol de alta densidade (HDL), fração de colesterol de baixa densidade (LDL), fração de colesterol de muito baixa densidade (VLDL) e glicose de jejum. As avaliações iniciais e finais foram realizadas em um mesmo laboratório de referência no município do Rio Grande/RS.

Todos os trabalhadores também responderam ao questionário para avaliação da qualidade de vida WHOQOL Bref. Este questionário, recomendado pela Organização Mundial de Saúde (OMS), é uma versão simplificada (que avalia as dimensões física, social, ambiental e psicológica) do questionário original WHOQOL e foi traduzido e validado no Brasil (FLECK et al., 1999). O domínio físico se focaliza nas seguintes facetas: dor e desconforto, energia e fadiga, sono e repouso, mobilidade, atividades da vida cotidiana, dependência de medicação ou de tratamentos, capacidade para o trabalho. No domínio psicológico, as facetas são: sentimentos positivos, pensar, aprender, memória, concentração, autoestima, imagem corporal e aparência, sentimentos negativos, espiritualidade, religiosidade e crenças pessoais. O domínio social inclui as seguintes facetas: relações pessoais, suporte (apoio) social, atividade sexual. Por fim, o domínio ambiental, abordando as facetas: segurança física e proteção, ambiente no lar, recursos financeiros, cuidados de saúde e sociais (disponibilidade e qualidade, oportunidade de adquirir novas informações e habilidades, participação e oportunidades de recreação/lazer) e ambiente físico (poluição, ruído, trânsito, clima e transporte).

Os trabalhadores realizaram ainda as seguintes avaliações: prova ergométrica, espirometria e avaliação alimentar recordatória de 24 horas, tendo sido realizada a avaliação de consumo e de gasto energético individuais.

Foi avaliado o aumento nos valores do consumo máximo de oxigênio ( $\mathrm{VO}_{2}$ máximo) atingido pelos trabalhadores no último estágio finalizado durante a prova ergométrica, classificados conforme The Cooper Institute for Aerobics Research (HEYWARD, 2006).

Os cálculos do equilíbrio energético foram realizados considerando-se as necessidades energéticas básicas de acordo com o peso, as atividades físicas referidas e os valores de consumo relatados no diário alimentar recordatório (Kcal/dia). Para determinação dos percentuais de gordura inicial e final, foi utilizado um adipômetro científico (Cescorf) para avaliar a espessura das dobras cutâneas e, posteriormente, utilizadas equações preditivas onde são consideradas as características biológicas e étnicas dos trabalhadores conforme descrito na literatura (HEYWARD; STOLARCZYK, 2000).
A partir da caracterização do perfil de saúde desses trabalhadores, o programa de promoção de saúde consistiu na proposição de atividade física e acompanhamento nutricional regulares. Além do monitoramento frequente do peso, os encontros semanais com a nutricionista proporcionavam um momento de discussão em grupo em que foram abordados diferentes temas relacionados à importância da adoção de hábitos alimentares saudáveis. Foi estimulada uma mudança gradual dos alimentos consumidos na embarcação, sendo realizado um trabalho mais intenso junto ao cozinheiro, uma vez que a compra e o preparo dos alimentos era de sua responsabilidade. Também foi realizada uma reunião com os familiares dos tripulantes visando mostrar a importância da mudança de hábitos alimentares da própria família, auxiliando no período em que os trabalhadores permaneciam em casa. Com exceção desta última atividade, que foi realizada em uma sala disponibilizada na própria estrutura portuária, as demais intervenções da nutricionista com a tripulação e com o cozinheiro foram desenvolvidas no refeitório da embarcação.

A intervenção física consistiu na proposição de 3 sessões semanais de atividade física, sempre com a orientação de um educador físico. Considerando que os trabalhadores passavam 3 dias consecutivos na embarcação, duas destas sessões eram realizadas a bordo. A outra sessão era realizada em uma academia ou eventualmente em outros lugares (praças públicas, rua, praia), sempre com o acompanhamento do educador físico. Na impossibilidade de comparecimento à academia, foi sugerida a realização de uma atividade de substituição em casa, como caminhada ou corrida leve.

Foi ainda disponibilizada na embarcação uma bicicleta ergométrica para realização de exercícios nos momentos em que não havia manobras no porto. $\mathrm{O}$ tempo de atividade na bicicleta era registrado pelo próprio trabalhador e o das demais atividades pelo educador físico, para posterior correlação com os resultados obtidos.

O efeito do tempo de atividades realizadas sobre alguns parâmetros da saúde dos trabalhadores foi analisado de duas formas. Inicialmente, foi contabilizado o tempo total de atividades (atividades desenvolvidas na embarcação, sob orientação do educador físico, e na bicicleta ergométrica, e atividades realizadas na academia - caminhadas/corridas em esteira e musculação). Também foi somado o tempo de caminhada realizada pelo trabalhador em casa, em substituição ao trabalho físico na academia. Em uma segunda análise, foram somados somente os tempos das atividades realizadas na embarcação com supervisão, ativi dades de caminhadas/corridas em esteira na academia e as caminhadas realizadas pelo trabalhador em casa.

Considerando as dificuldades que podem representar mudanças de hábitos e estilos de vida, este programa 
previu a participação de um profissional especializado em motivação. Este profissional realizou dois encontros com o grupo de trabalhadores, no início e ao final das primeiras 11 semanas de intervenção. Essas intervenções foram realizadas inicialmente com o objetivo de estimular a adesão do grupo ao programa e, em um segundo momento, sua perseverança nas diferentes atividades propostas (2 horas com cada tripulação). Durante esses encontros, realizados no interior do próprio rebocador, foram utilizadas as técnicas de "Teoria das Escolhas" e "Conceito de Mapas Pessoais" (GLASSER, 2001; CHUNG, 1995). A avaliação da intervenção motivacional foi realizada de forma quantitativa, solicitando aos trabalhadores que atribuíssem uma nota de 1 a 10 para expressar a vontade de envolvimento total com o programa.

O projeto foi submetido ao Comitê de Ética em Pesquisa na Área da Saúde da Universidade Federal do Rio Grande (parecer no 97/2009) e por ele aprovado, e a participação dos trabalhadores deu-se após a assinatura do termo de consentimento livre e esclarecido. Devido à natureza interdisciplinar da proposta, a equipe de pesquisa foi formada por duas fisioterapeutas, uma enfermeira, uma nutricionista, um educador físico, um médico e por um profissional especializado em motivação em grupo.

Para análise dos dados, foi realizada uma distribuição de frequência das variáveis categóricas e calculada a média (desvio padrão) das variáveis contínuas. Para a comparação dos diferentes parâmetros analisados antes e depois da intervenção, foi utilizado o teste $\mathrm{t}$ pareado. O teste de Wilcoxon foi utilizado para análise dos domínios da qualidade de vida. Para análise de correlação dos dados foi utilizado o teste de Pearson. Foram considerados significativos valores em que $\mathrm{p}<0,05$.

\section{Resultados}

Todos os trabalhadores que fizeram parte do projeto eram do sexo masculino. A média de idade do grupo foi de 44,0 \pm 7,9 anos.

O peso dos trabalhadores reduziu significante mente entre a avaliação inicial e final ( $p=0,03)$.No entanto, o IMC do grupo não foi modificado de forma significativa. As medidas da CA ( $p=0,005)$ e da CQ ( $p=0,005)$ foram reduzidas de forma significativa, refletindo-se em uma redução no ICQ, mesmo que essa não tenha sido significativa (Tabela 1).

O tempo médio de exercícios realizados pelos trabalhadores foi de 86,8 \pm 22,2 minutos/semana. Desses, 42,1 \pm 12,6 minutos/semana foram atividades desenvolvidas no barco e caminhadas/corridas na esteira da academia sob a supervisão do educador físico mais as caminhadas realizadas em casa em substituição à atividade da academia. Foi verificado que este último valor (somatório do tempo de atividades sob orientação na embarcação e na esteira na academia, mais caminhadas em casa) se correlacionou positivamente com a redução da CA ( $\mathrm{r}=0,636$; $\mathrm{p}=0,048)$ e do ICQ ( $\mathrm{r}=0,683 ; \mathrm{p}=0,03)$.

A avaliação bioquímica realizada antes e depois do programa piloto não mostrou diferença significativa quanto aos níveis de glicose no sangue dos traba-

Tabela 1 Valores médios \pm desvio padrão de peso, índice de massa corporal (IMC), circunferência abdominal (CA), circunferência do quadril (CQ), índice cintura-quadril (ICQ) e dos diferentes domínios que compõem a avaliação da qualidade de vida de trabalhadores marítimos de rebocadores $(n=10)$ antes e depois de programa de promoção da saúde, Rio Grande/RS, junho de 2010

\begin{tabular}{lccc}
\hline & Inicial & Final & $p$ \\
\hline Peso $(\mathrm{kg})$ & $84,3 \pm 14,1$ & $82,1 \pm 14,9$ & $0,03^{*}$ \\
IMC $\left(\mathrm{kg} / \mathrm{m}^{2}\right)$ & $28,1 \pm 4,1$ & $27,6 \pm 4,1$ & 0,18 \\
CA $(\mathrm{cm})$ & $97,1 \pm 12,7$ & $91,7 \pm 12,6$ & $0,005^{*}$ \\
CQ $(\mathrm{cm})$ & $103,5 \pm 5,4$ & $100,7 \pm 7,1$ & $0,005^{*}$ \\
ICQ $(\mathrm{cm})$ & $0,93 \pm 0,01$ & $0,91 \pm 0,01$ & 0,09 \\
Domínio físico & $78,6 \pm 13,3$ & $83,6 \pm 11,9$ & 0,16 \\
Domínio psicológico & $76,1 \pm 8,8$ & $77,5 \pm 9,9$ & 0,63 \\
Domínio social & $84,2 \pm 14,9$ & $78,3 \pm 8,1$ & 0,30 \\
Domínio ambiental & $61,2 \pm 9,3$ & $64,7 \pm 10,3$ & 0,11 \\
\hline
\end{tabular}

* significante $(p<0,05)$ 
lhadores. Os valores médios de HDL foram reduzidos (53,3 x 47,7 mg/dl; p = 0,02). Após a participação no programa, houve uma redução significativa $(\mathrm{p}=0,04)$ no número de marítimos com triglicerídeos elevados ( $\geq 170 \mathrm{mg} / \mathrm{dl}$ ), passando de 7 para 3 os trabalhadores nesta condição. A redução observada quanto aos demais lipídeos não foi significativa (Figura 1).

No que se refere à avaliação da qualidade de vida, não foi observada diferença significativa entre o início e o final do programa de intervenção. Na avaliação final, o domínio que recebeu maior nota foi o domínio físico e a menor nota foi atribuída ao domínio ambiental (Tabela 1).

O percentual médio de gordura do grupo de trabalhadores que participou da intervenção reduziu significativamente após a intervenção (inicial 23,0\%; final 21,4\%; p < 0,00). A análise da avaliação das pregas cutâneas mostrou que 2 dos 5 trabalhadores com porcentagem de gordura considerada acima do normal atingiram a condição de normalidade após a participação no programa (Tabela 2).

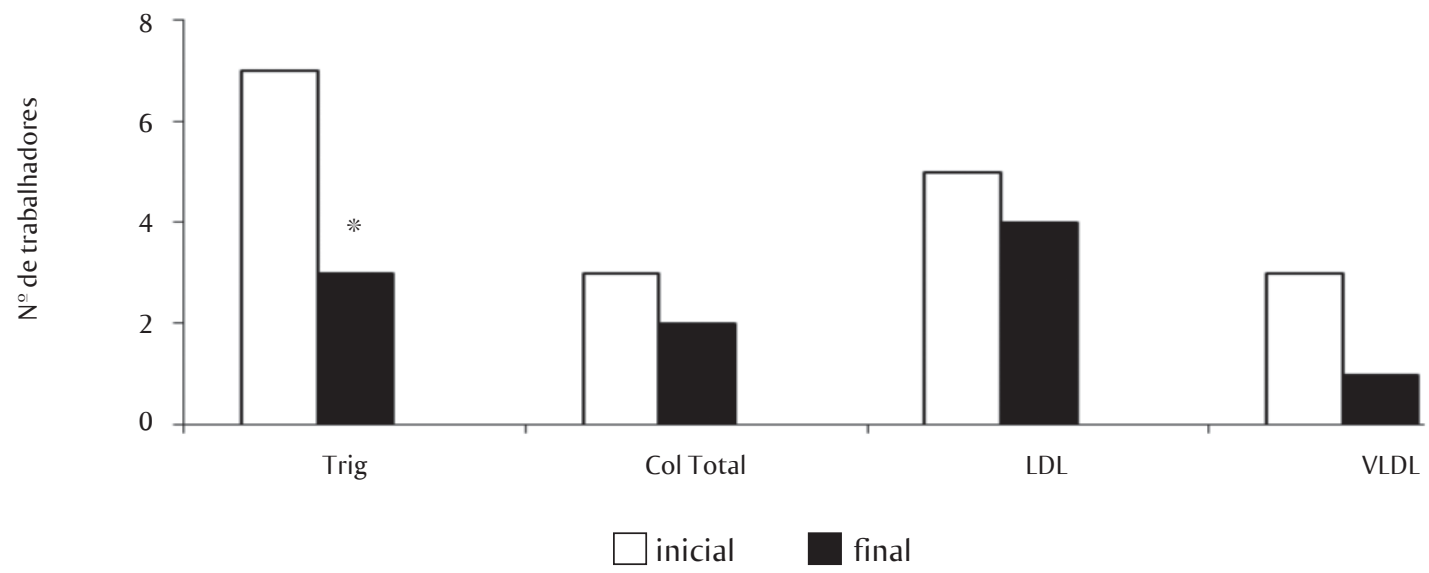

Figura 1 Alterações em lipídeos**** de trabalhadores marítimos de rebocadores ( $n=10)$ antes e depois da participação em programa piloto de promoção da saúde. Rio Grande, RS, 2010

* diferença significativa $(\mathrm{p}<0,05)$.

*** Trig = triglicérides; $\mathrm{Col}=$ colesterol total; $\mathrm{LDL}=$ colesterol de baixa densidade; $\mathrm{VLDL}=$ colesterol de muito baixa densidade.

Tabela 2 Percentual de gordura e da classificação do $\mathrm{VO}_{2}$ máximo dos trabalhadores marítimos de rebocadores $(\mathrm{n}=10)$ antes e depois do programa de promoção da saúde, Rio Grande, RS, 2010

\begin{tabular}{lcc}
\hline & Inicial & Final \\
& $\mathrm{n}$ & $\mathrm{n}$ \\
\hline \% de Gordura & & \\
Normal & 01 & 03 \\
Baixo & 01 & 01 \\
Acima & 05 & 03 \\
Muito Acima & 03 & 03 \\
& & \\
Classificação do $\mathrm{VO}_{2}{ }^{*}$ & & 05 \\
Excelente & 02 & 01 \\
Muito Bom & 02 & 03 \\
Bom & - & - \\
Regular & 03 & 01 \\
Ruim & 03 & \\
\hline
\end{tabular}

*Conforme The Cooper Institute for Aerobics Research (HEYWARD, 2006). 
A comparação do cálculo do equilíbrio energético antes e após a intervenção mostrou uma redução significativa $(\mathrm{p}=0,03)$ no consumo calórico diário, passando de 3.061,5 \pm 597,5 kcal na primeira avaliação para $2.537,5 \pm 210,5 \mathrm{kcal}$ ao final das 22 semanas de intervenção.

A análise ergométrica final mostrou que, após o período de intervenção, a média do $\mathrm{VO}_{2}$ máximo dos trabalhadores aumentou de forma significativa

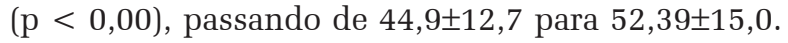
A evolução da classificação dos trabalhadores de acordo com a sua capacidade cardiorrespiratória pode ser observada na Tabela 2.

A totalidade dos trabalhadores do grupo intervenção apresentou espirometria dentro da normalidade antes e após a intervenção.

Nos dois momentos de atividades de motivação realizadas (início e metade do período de intervenção), $80 \%$ dos trabalhadores atribuíram nota 10 (1 a 10) quanto ao seu desejo de envolvimento com o projeto.

\section{Discussão}

A população alvo deste estudo piloto possui peculiaridades no que diz respeito à sua heterogeneidade demográfica, de condicionamento físico e de diversidade de atividades, pois o grupo avaliado era composto por condutores mestres de embarcação, homens encarregados das máquinas e homens de atividade em convés. Além disso, os trabalhadores marítimos de rebocadores enfrentam outros desafios que independem da atividade específica desenvolvida, como longos turnos de trabalho e alternância de períodos de estresse elevado e sedentarismo.

Os trabalhadores incluídos neste estudo e submetidos ao programa de promoção da saúde, baseado em intervenção nutricional educativa e estímulo à prática de atividade física, apresentavam um perfil de saúde marcado pela obesidade e por dislipidemias (BAENA et al., 2010). Dos 10 trabalhadores que participaram do programa, 8 apresentavam a condição de sobrepeso ou obesidade. Os trabalhadores mostraram uma redução significativa do peso e do percentual médio de gordura após a intervenção. Porém, não foi observada mudança significativa no IMC.

Como não são encontrados estudos sobre participação de trabalhadores de rebocadores em programas de promoção da saúde, o presente estudo piloto trouxe informações valiosas sobre a evolução do perfil de saúde destes trabalhadores marítimos com características tão específicas, submetidos a este modelo de intervenção.
Ciorlia e Godoy (2005), num estudo com eletricitários baseado em um programa preventivo de riscos cardiovasculares realizado pela medicina ocupacional de uma empresa paulista, mostraram que, mesmo com orientação dietética e estímulo à prática de atividade física durante um ano, houve aumento gradual e significativo do IMC dos trabalhadores estudados. Em uma empresa do ramo alimentício, um programa de promoção de saúde com duração de 12 meses, que previa incentivo à prática de atividades físicas e orientação nutricional, resultou em aumento da atividade física sem alteração significativa do IMC (BANDINI, 2006). Em ambos os estudos, o curto tempo de acompanhamento é sugerido como hipótese para a não tradução da perda de peso em diminuição do IMC. Da mesma forma, acredita-se que o tempo de acompanhamento deste estudo piloto tenha sido uma limitação no sentido de modificação significativa do IMC.

A significativa diminuição dos valores de CA entre os trabalhadores deste estudo representa um dado importante, uma vez que esta medida tem se apresentado como o indicador antropométrico mais fortemente correlacionado com maior número de variáveis de risco cardiovascular, como triglicerídeos elevados, HDL baixo, partículas de LDL subclassificadas como pequenas e densas, hiperlipidemia pós-prandial, resistência à insulina e hipertensão arterial sistêmica, condições que em conjunto caracterizam a síndrome metabólica (REZENDE et al., 2006; SPOSITO et al., 2007). Esta redução na CA esteve acompanhada de uma redução significativa da CQ, embora não tenha sido verificada significação na redução do ICQ. Resultados semelhantes foram encontrados por outros autores em estudo realizado com um grupo de indivíduos obesos e hipertensos submetidos a um programa de orientação nutricional (ALVAREZ; ZANELLA, 2009).

O resultado positivo sobre os índices antropométricos (peso, circunferência abdominal e do quadril) poderiam ser explicados em parte pela adesão dos trabalhadores às atividades físicas propostas. Foi verificado que o somatório do tempo de atividade física realizada pelos trabalhadores, na academia ou na própria embarcação, mostrou correlação positiva significativa com a redução da CA e redução no ICQ. Efeito semelhante foi observado em outro estudo em que os indivíduos que realizavam maior tempo de atividade física semanal apresentaram menor armazenamento de gorduras na região central e maior controle dos lipídeos plasmáticos (CHEIK et al., 2006).

Somando-se ao efeito da atividade física, destaca-se também a importância da orientação nutri- 
cional para os efeitos encontrados, sobretudo no que se refere ao acompanhamento do cozinheiro da embarcação. A orientação do nutricionista na compra de alimentos mais saudáveis, na escolha de cardápios mais equilibrados e no preparo dos alimentos de uma maneira mais adequada parece ser uma estratégia que também contribui para os resultados encontrados.

A obesidade central tem sido fortemente associada com a idade, o sexo feminino e o sedentarismo segundo Martins e Marinho (2003). Esses autores verificaram que os indivíduos que realizam atividade física leve têm o dobro de chances de desenvolver a obesidade abdominal em relação aos que realizam atividade física moderada. Em um estudo de revisão sobre os efeitos do tempo e da intensidade das atividades físicas foi enfatizado que a recomendação de realização de atividade de moderada a grande intensidade por 30 minutos, de preferência todos os dias, mesmo que eficaz na prevenção da doença cardiovascular, parece ser insuficiente para a prevenção de ganho de peso em alguns indivíduos. Além disso, para evitar que indivíduos que já foram obesos readquiram excesso de peso, faz-se necessária a realização de atividade diária moderada por 60 a 90 minutos ou tempo menor, se a atividade desenvolvida for de grande intensidade (SARIS et al., 2003).

Em nosso estudo, a correlação verificada entre a redução da CA e do ICQ e o tempo de atividade desenvolvida reforça o que já vem sendo apontado por outros autores sobre a importância de uma atividade física moderada para a redução da gordura abdominal (KAY; SINGH, 2006).

Ao mesmo tempo, os resultados positivos sobre a antropometria também podem ser explicados por uma redução significativa no consumo médio diário de calorias, observado ao término da intervenção. A orientação nutricional semanal proposta no programa, o trabalho educativo intenso realizado junto ao cozinheiro da embarcação e a troca gradual dos alimentos estimulando o consumo, por parte dos trabalhadores, de uma alimentação mais nutritiva e com menor valor calórico certamente contribuíram para os resultados obtidos.

Os dados deste estudo mostram uma redução significativa na concentração de triglicerídeos no sangue de 4 dos 7 trabalhadores que apresentavam este tipo de dislipidemia, sem redução significativa quanto ao colesterol total, LDL e VLDL. Os organizadores da IV Diretriz Brasileira Sobre Dislipidemias e Prevenção da Aterosclerose enfatizam que a prática de exercícios físicos aeróbios se constitui em uma medida auxiliar para o controle das dislipidemias, promovendo a redução de triglicerídeos, porém sem alterações significativas sobre as concentrações de LDL (SPOSITO et al., 2007).

O aumento na atividade física entre os trabalhadores foi acompanhado de uma redução significativa nos níveis de HDL. A prática de atividade física tem sido associada ao aumento do HDL (SPOSITO et al., 2007; PITANGA, 2001). No entanto, também tem sido sugerido que o HDL pode reduzir transitoriamente durante a fase inicial de perda de peso em indivíduos obesos quando são adotadas dietas hipolipídicas, o que parece desaparecer com a continuidade da dieta ou quando é atingida a situação de eutrofia (NONAS, 1998). Acredita-se que o tempo de acompanhamento deste estudo piloto talvez não tenha sido suficiente para se observar uma situação de eutrofia na qual se pudesse verificar um nivelamento do HDL sanguíneo.

Um dos resultados mais expressivos encontrados após a intervenção se refira à melhora da capacidade cardiorrespiratória dos trabalhadores observada pelo aumento no seu $\mathrm{VO}_{2}$ máximo. Estudos recentes mostram que cada MET ganho (equivalente metabólico que expressa o consumo de oxigênio e que pode ser definido pela razão entre a taxa metabólica durante uma atividade física específica e uma taxa de referência da taxa metabólica em repouso) pode significar uma redução de 15\% no risco cardiovascular (HEYWARD, 2006). O ganho de $\mathrm{VO}_{2}$ médio observado no conjunto de trabalhadores sugere uma redução no risco de eventos cardiovasculares entre esses trabalhadores.

Outra manifestação desta melhora de aptidão física é que, por vontade dos trabalhadores e com orientação do profissional de educação física, 7 dos 10 trabalhadores do grupo de intervenção iniciaram a prática de corridas de rua. Três deles participaram de 3 corridas de $10 \mathrm{~km}$ durante o programa.

Mesmo com os resultados positivos obtidos no que se refere aos índices antropométricos, à redução da concentração de triglicerídeos sanguíneos e ao aparente envolvimento dos trabalhadores verificado pela nota atribuída pelos mesmos quanto à sua motivação, não foi observada diferença significativa após a intervenção em relação a nenhum dos domínios que constituem a avaliação de qualidade de vida. A não tradução dos resultados obtidos e das avaliações qualitativas positivas realizadas pelos trabalhadores ao longo do programa em índices significativos no instrumento de qualidade de vida chama a atenção. É possível que, ao final do período de intervenção, os trabalhadores tenham se sentido mais confiantes para expressar a sua opinião real sobre cada um dos temas investigados. No entanto, a melhora dos parâmetros de saúde sem que haja melhora na avaliação da qualidade de vida foi também observada por Bandini (2006). 
Não foram encontrados estudos de intervenções nutricional e física realizadas no local de trabalho de indivíduos com turnos tão específicos (3 dias e 4 noites) para comparação de resultados. Este fato reforça a importância da apresentação dos resultados obtidos neste programa piloto, mesmo com o número reduzido de participantes.

No que se refere à intervenção nutricional, os resultados parciais obtidos em relação à bioquímica sanguínea dos trabalhadores ao final da intervenção apontam para a necessidade de uma abordagem mais intensa junto às famílias, o que poderia contribuir para resultados mais positivos em relação a mudanças de hábitos entre esses trabalhadores com especificidades ocupacionais tão peculiares. Conforme relato dos próprios trabalhadores, quando do retorno para casa após um afastamento pelo turno de trabalho de 72 horas, frequentemente, a família utiliza a alimentação como forma de compensação durante sua permanência no lar. A contribuição familiar na adesão a um programa de mudanças de estilo de vida foi determinante em estudo realizado por Fechio e Malerbi (2004) com indivíduos diabéticos. Os autores relatam que os indivíduos que tiveram maior adesão foram aqueles cujo membro da família também aderiu ao programa.

Os resultados obtidos com a aplicação deste programa piloto reforçam a importância das intervenções nutricional e física conjuntas apontando também para a necessidade de ampliar o tempo de desenvolvimento do programa de forma a despertar entre os trabalhadores modificações contínuas e duradouras em seus hábitos alimentares e quanto à prática de atividade física regular. Para tanto, os programas de promoção de saúde devem estar de acordo com as recomendações da Organização Pan-Americana da Saúde, segundo as quais as intervenções devem considerar o contexto social e as necessidades dos diferentes grupos de população (PAN AMERICAN HEALTH ORGANIZATION, 2002).

\section{Considerações finais}

Este artigo apresentou os efeitos de um programa piloto de promoção da saúde sobre algumas características biológicas e aspectos da qualidade de vida de um grupo de trabalhadores muito pouco estudados, os marítimos de rebocadores.

Alguns princípios da promoção de saúde, como o estímulo à adoção de uma alimentação equilibrada e à realização regular de atividade física, devem nortear as práticas relacionadas à saúde dos marítimos de rebocadores. E este aspecto torna-se ainda mais relevante no caso dessa categoria de trabalhadores que enfrenta turnos de trabalho longos e situações de estresse elevado alternados com períodos de sedentarismo.

Consideradas as limitações decorrentes do número restrito de participantes, os efeitos positivos observados neste estudo em parâmetros como peso, circunferência abdominal e do quadril, triglicerídeos e $\mathrm{VO}_{2}$ máximo indicam a atuação deste programa avaliado na redução do risco de morbidade e mortalidade por doenças cardiovasculares entre esses trabalhadores. Outros estudos são necessários para avaliar a efetividade dessa intervenção e seus benefícios para um grupo maior de trabalhadores marítimos de rebocadores, com um tempo maior de acompanhamento.

A partir dessa análise inicial, verificou-se a necessidade de alguns ajustes metodológicos como tempo de intervenção e maior envolvimento da família. Considerando-se as características peculiares do trabalho dos marítimos de rebocadores, recomenda-se a continuidade no acompanhamento destes trabalhadores e uma avaliação do impacto a médio e longo prazos das mudanças observadas sobre a sua saúde e sua qualidade de vida.

\section{Contribuições de autoria}

Todos os autores tiveram participação ativa em todas as fases do estudo.

\section{Referências}

ALVAREZ, T. S.; ZANELLA, M. T. Impacto de dois programas de educação nutricional sobre o risco cardiovascular em pacientes hipertensos e com excesso de peso. Revista de Nutrição, Campinas, v. 22, n. 1, p. 71-79, jan./fev. 2009.

BAENA, C. P. et al. Perfil de saúde dos trabalhadores marítimos de rebocadores que participaram de um programa de promoção da saúde. Revista Vittalle, Rio Grande, v. 22, n. 1, p. 65-74, 2010.

BANDINI, M. Impacto de ações de promoção da saúde incluídas no Programa de Controle Médico de Saúde Ocupacional de empresa do ramo alimentício. 2006. 246 f. Tese (Doutorado em Ciências - Patologia)-Faculdade de Medicina, Universidade de São Paulo, São Paulo, 2006. 
BUSS, P. M. Promoção da saúde e qualidade de vida. Ciência \& Saúde Coletiva, Rio de Janeiro, v. 5, n. 1, p. 163-177, 2000.

CIORLIA, L. A. S.; GODOY, M. F. Fatores de risco cardiovascular e mortalidade. Seguimento em longo prazo (até 20 anos) em programa preventivo realizado pela medicina ocupacional. Arquivos Brasileiros de Cardiologia, Rio de Janeiro, v. 85, n. 1, p. 20-25, jul. 2005.

CHAPMAN, L. S. Proof Positive. An analysis of the cost effectiveness of worksite wellness. Seattle: Summex Corporation, 2002.

CHEIK, N. C. et al. Efeito de diferentes freqüências de exercício físico na prevenção da dislipidemia e da obesidade em ratos normo e hipercolesterolêmicos. Revista Brasileira de Educação Física e Esporte, São Paulo, v. 20, n. 2, p. 121-129, jun. 2006.

CHUNG, T. Qualidade começa em mim: manual neurolinguístico de liderança e comunicação. São Paulo: Maltese, 1995.

FECHIO, J. J.; MALERBI, F. E. K. Adesão a um programa de atividade física em adultos portadores de diabetes. Arquivos Brasileiros de Endocrinologia $\mathcal{\sigma}$ Metabolismo, São Paulo, v. 48, n. 2, p. 267-275, abr. 2004.

FLECK, M. et al. Aplicação da versão em português do instrumento de avaliação de qualidade de vida da Organização Mundial da Saúde (WHOQOL-100). Revista de Saúde Pública, São Paulo, v. 33, n. 2, p. 198-205, abr. 1999.

GLASSER, W. Teoria da Escolha: uma nova psicologia de liberdade pessoal. São Paulo: Mercuryo, 2001.

GRAÇA, L. A Promoção de saúde no trabalho: a nova saúde ocupacional? Lisboa: Sociedade Portuguesa de Medicina do Trabalho, 1999. (Cadernos Avulsos, 1). Disponível em: < http://www.ensp.unl.pt/lgraca/ textos72.html>. Acesso em: 25 jul. 2010.

HEYWARD, V. H. Advanced fitness assessment and exercise prescription. Champaign, IL: Human Kinetics, 2006.

HEYWARD, V. H.; STOLARCZYK, L. M. Avaliação da composição corporal aplicada. São Paulo: Manole, 2000.

INSTITUTO BRASILEIRO DE GEOGRAFIA E ESTATÍSTICA. Tabulação avançada do Censo Demográfico 2000. Rio de Janeiro, 2002.

KAY, S. J.; SINGH, M. A. F. The influence of physical activity on abdominal fat: a systematic review of the literature. Obesity Reviews, Oxford, v. 7, n. 2, p. 183-200, mai. 2006.
MARTINS, I. S.; MARINHO, S. P. O potencial diagnóstico dos indicadores da obesidade centralizada. Revista de Saúde Pública, São Paulo, v. 37, n. 6, p. 760767, dez. 2003.

MENDONÇA, C. P.; ANJOS, L. A. Aspectos das práticas alimentares e da atividade física como determinantes do crescimento do sobrepeso/obesidade no Brasil. Cadernos de Saúde Pública, Rio de Janeiro, v. 20, n. 3, p. 698-709, maio/jun. 2004.

NONAS, C. A. A model for chronic care of obesity through dietary treatment. Journal of the American Dietetic Association, United States, v. 98, n. 10 (Suppl.), p. S16-S22, oct. 1998.

OGATA, A. A obesidade é um problema para as empresas? Biblioteca Sebrae, 2007. Disponível em: <http://www.biblioteca.sebrae.com.br/bds/BDS. nsf/7ff4db7574e364c80 3256ebc004add8c/2d62b3d53 b9d2a7a03256f97004112ef/\$FILE/NT000A35F6.pdf > . Acesso em: 01 fev. 2010.

PAN AMERICAN HEALTH ORGANIZATION. Public Health Response to Chronic Diseases. Washington (DC): PAHO, 2002.

PITANGA, F. J. G. Atividade física e lipoproteínas plasmáticas em adultos de ambos os sexos. Revista Brasileira de Ciência e Movimento, Brasília, v. 9, n. 4, p. 25-31, out/dez. 2001.

REZENDE, F. A. C. et al. Índice de massa corporal e circunferência abdominal: associação com fatores de risco cardiovascular. Arquivos Brasileiros de Cardiologia, Rio de Janeiro, v. 87, n. 6, p. 728-734, dez. 2006.

ROBERTS, S. E. Mortality from disease among seafarers in British merchant shipping (1976-1995). International Maritime Health, Poland, v. 53, n. 1-4, p. 43-58, 2003.

SARIS, W. H. et al. How much physical activity is enough to prevent unhealthy weight gain? Outcome of the IASO 1st Stock Conference and Consensus Statement. Obesity Review, v. 4, p. 101-114, 2003.

SPOSITO, A. C. et al. Quarta diretriz brasileira sobre dislipidemias e prevenção da aterosclerose: Departamento de Aterosclerose da Sociedade Brasileira de Cardiologia. Arquivos Brasileiros de Cardiologia, São Paulo, v. 89, n. 4 (Supl. 1), p. 2-19, 2007.

WORLD HEALTH ORGANIZATION. Obesity: preventing and managing the global epidemic. Geneva, 2000. 